\title{
Frequency Deviation of Injection-Locked Microwave Autodynes
}

\author{
Vladislav Ya. NOSKOV, Kirill A. IGNATKOV, Kirill D. SHAIDUROV \\ Dept. of Radioelectronics and Telecommunications, Ural Federal University (UrFU), \\ Mira St., 19, 620002, Yekaterinburg, Russia \\ noskov@oko-ek.ru
}

Submitted June 1, 2019 / Accepted August 28, 2019

\begin{abstract}
This article presents the results of the autodyne (AD) frequency deviation research, which is simultaneously affected by its own reflected radiation and the external synchronization signal. The basic correlations are obtained for the injection-locked AD (ILAD) analysis in the case of a moving radar object. The amplitude-frequency characteristics are calculated for the transfer coefficient of the autodyne phase variations and the ILAD frequency deviation. The advantages of ILAD are shown compared to $A D$ without synchronization. The theoretical research investigations are confirmed by experimental data obtained by the example of an oscillator made on the basis of the 8-mm Gunn diode.
\end{abstract}

\section{Keywords}

Autodyne, injection-locked autodyne, autodyne signals, frequency deviation, Gunn-diode oscillator

\section{Introduction}

Virtually, any microwave oscillators supplemented by appropriate autodyne response recording means can be used as autodynes ${ }^{1}$ (AD). At the same time, they combine the functions of a probe transmitter and a highly sensitive receiver of its own reflected microwave radiation from the radar target. Owing to the constructive simplicity and low cost, such transceiver units are widely used as radar sensors in transport, industry, research, military purposes, and medicine [8], [10-17]. Therefore, the interest to studying

The terms like oscillator-detector [1], self-oscillating mixer (SOM) [2], self-mixing oscillator [3], self-detecting oscillator [4], selfexcited mixer [5], load variation detector [5], [6], homodyne detector [7] etc. are often used along with the widespread term of "autodyne" in the reference sources. In this paper, we consider the autodyne as the radar device [8]. The operation principle and the behavior of such autodynes, which are at the action of the own reflected emission significantly differs from autodynes, which fulfill the functions of signal frequency conversion obtained from the external independent source [9]. of $\mathrm{AD}$ parameters and characteristics, to investigations of the new types of oscillators and their operation modes, as well as to expansion of $\mathrm{AD}$ application area is steadily increasing.

One of the most important parameters of both radio frequency and laser $\mathrm{AD}$ as well as $\mathrm{AD}$ with various types of modulation is the so-called feedback parameter (FBP), $C_{\mathrm{FB}}=\Delta \omega_{\mathrm{AD}} \tau$, where $\omega_{\mathrm{AD}}$ is the autodyne frequency deviation value; $\tau$ is the delay time of the reflected microwave radiation [15], [18-20]. The physical meaning of this parameter FBP is the phase modulation index of the autodyne response. This modulation is caused by autodyne variations in the generation frequency when exposed to a reflected radiation oscillator. The value of this parameter determines the degree of anharmonic distortions of autodyne signals, which create serious problems in its processing, especially with the growth of the AD operating frequency.

It should be noted that the value of the $C_{\mathrm{FB}}$ parameter is significantly influenced by the distance to the radar object. This influence has the direct influence through the time lag parameter $\tau$, and it acts indirectly through the autodyne frequency deviation parameter $\Delta \omega_{\mathrm{AD}}$ since $\Delta \omega_{\mathrm{AD}}$ depends on the level of microwave radiation reflected from the radar object and on the distance, respectively. Therefore, the main way to reduce signal distortions is to reduce the autodyne frequency deviation of the used microwave oscillator. Various types of oscillators are studied on this way: multi-frequency [21], [22] and multi-element [23], [24] as well as stabilized by means of an additional highquality cavity [25], [26].

There are also publications that present the results of the injection-locked AD (ILAD) research [8], [27-29]. However, the problem of possible distortions of ILAD signals due to autodyne variations in the generation frequency was not considered in known reference sources. Nevertheless, the lack of any research results concerning this phenomenon impedes a deeper understanding of the processes in the system and, thus, it hinders the further development of this area and the practical use of ILAD in the future short-range radar systems. 
In this regard, the purpose of this paper is to study the autodyne frequency deviation of ILAD at both low and high speeds of movement of radar objects depending on the level of reflected radiation, the internal parameters of the oscillator used and its synchronization conditions.

\section{The Main Relations for the ILAD Analysis}

To obtain the mathematical model of ILAD, we consider the equivalent circuit presented in Fig. 1. In this circuit, $Y_{\mathrm{AE}}$ depicts the average (over the oscillation period) conductance of the active element (AE) of the oscillator, which in the general case depends on the amplitude $A$ and the current frequency $\omega$ of oscillation: $Y_{\mathrm{AE}}=G_{\mathrm{AE}}+\mathrm{j} B_{\mathrm{AE}}=$ $G_{\mathrm{AE}}(A, \omega)+\mathrm{j} B_{\mathrm{AE}}(A, \omega)$, where $G_{\mathrm{AE}}, B_{\mathrm{AE}}$ are resistive and reactive conductance of $\mathrm{AE}$, respectively. $Y_{\mathrm{OS}} \equiv Y_{\mathrm{OS}}(\omega)$ is the conductance of the oscillating system (OS) reduced to the AE plane. The equivalent current sources $j_{\text {ex }}(t)$ and $j_{\text {own }}(t, \tau)$ depict, respectively, 'external' impact on ILAD of the harmonic signal and the own emission delayed on time $\tau$ and reflected from the target: $j_{\mathrm{ex}}(t)=\operatorname{Re}\left[J_{\mathrm{ex}} \exp \left(\mathrm{j} \omega_{\mathrm{ex}} t\right)\right]$, $j_{\text {own }}(t, \tau)=\operatorname{Re}\left\{J_{\text {own }} \exp \left(\mathrm{j}\left[\omega_{\text {ex }} t+\varphi-\delta(t, \tau)\right]\right)\right\}$, where $J_{\text {ex }}$, $J_{\text {own }}$ are its amplitudes, $\omega_{\text {ex }}$ is the frequency; $\delta(t, \tau)$ is the phase incursion of the own reflected microwave radiation.

We suppose that under condition of the enough high Q-factor $Q_{\mathrm{L}}>>1$ of OS and at fulfillment of the phase and amplitude balance in the circuit, the quasi-harmonic oscillation acts on AE: $\dot{A}=A \exp \left[\mathrm{j}\left(\omega_{\mathrm{ex}} t+\varphi\right)\right]$, where $A=A(t)$, $\varphi=\varphi(t)$ are amplitude and phase, which are slowly varying over the oscillation period. Then, on the base of Kirchhoff laws for the considered circuit, we have the initial expression for analysis in the form:

$$
\operatorname{Re}\left[\dot{A} \times\left(Y_{\mathrm{AE}}+Y_{\mathrm{OS}}\right)\right]=j_{\text {own }}(t, \tau)+j_{\mathrm{ex}}(t) .
$$

Let us consider below the case of a single-circuit OS, for which the conductivity expression considering the load takes the form as follows:

$$
Y_{\mathrm{OS}}=G_{\mathrm{OS}}+\mathrm{j} B_{\mathrm{OS}}=G_{\mathrm{OS}}\left[1+\mathrm{j} 2 Q_{\mathrm{L}}\left(\omega-\omega_{\text {own }}\right) / \omega_{\text {own }}\right]
$$

where $\quad B_{\mathrm{OS}}=2 G_{\mathrm{OS}} 2 Q_{\mathrm{L}}\left(\omega-\omega_{\text {own }}\right) / \omega_{\text {own }}, \quad G_{\mathrm{OS}}=G_{\mathrm{own}}+G_{\mathrm{L}}$ are the reactive and resistive conductivity of OS; $G_{\text {own }}, G_{\mathrm{L}}$ are the conductivity of own losses of cavity and load respectively; $\omega_{\text {own }}$ is the natural frequency of OS.

Following the slow-changing amplitudes method [30], after substituting (2) into (1) and averaging the instan-

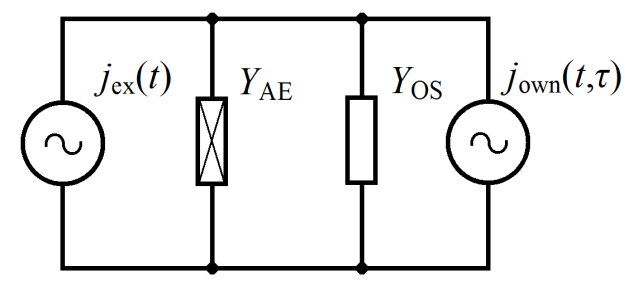

Fig. 1. Equivalent circuit of the injection-locked autodyne. taneous values over the oscillation period of ILAD, we obtain the abbreviated equations for the amplitude $A(t)$ and phase $\varphi(t)$ of the oscillations in the form as follows:

$$
\begin{gathered}
\frac{Q_{\mathrm{L}}}{\omega_{\mathrm{own}}} \frac{1}{A} \frac{\mathrm{d} A}{\mathrm{~d} t}+\frac{G_{\mathrm{AE}}+G_{\mathrm{OS}}}{2 G_{\mathrm{OS}}}=\Gamma \eta \cos \delta(t, \tau)+k_{\mathrm{ex}} \eta \cos \varphi, \\
\frac{Q_{\mathrm{L}}}{\omega_{\mathrm{own}}} \frac{\mathrm{d} \varphi}{\mathrm{d} t}+\frac{B_{\mathrm{AE}}+B_{\mathrm{OS}}}{2 G_{\mathrm{OS}}}=-\Gamma \eta \sin \delta(t, \tau)-k_{\mathrm{ex}} \eta \sin \varphi
\end{gathered}
$$

where $\Gamma=A_{\text {ref }} / A$ and $k_{\text {ex }}=A_{\text {ex }} / A$ are the coefficients characterizing the relative levels of the amplitudes of the reflected radiation $A_{\text {ref, }}$ and external synchronizing effects $A_{\text {ex }}=J_{\text {ex }} / G_{\mathrm{OS}} ; \eta=Q_{\mathrm{L}} / Q_{\mathrm{ex}}, Q_{\mathrm{ex}}$ are the efficiency induced by the microwave oscillator load and OS Q-factor, respectively.

The system of non-linear equations (3), (4) was obtained in the usual approximations [30] used for the analysis of self-oscillating systems, and has sufficient generality to describe transients and steady-state amplitudes and phases of oscillations of a microwave oscillator under the simultaneous or separate effects of oscillations from an external oscillator and (or) reflected radiation. In the case of the lack of radiation reflected from the radar object, when $\Gamma=0$, from the equations, we obtain the expressions for the steady-state values of the amplitude $A^{0}$ loc and phase $\varphi_{0}$ of the ILAD oscillations in the form as follows:

$$
\begin{aligned}
& {\left[G_{\mathrm{AE}}\left(A_{\mathrm{loc}}^{0}, \omega_{\mathrm{ex}}\right)+G_{\mathrm{OS}}\left(\omega_{\mathrm{ex}}\right)\right] /\left(2 G_{\mathrm{OS}}\right)=k_{\mathrm{ex}} \eta \cos \varphi_{0},} \\
& {\left[B_{\mathrm{AE}}\left(A_{\mathrm{loc}}^{0}, \omega_{\mathrm{ex}}\right)+B_{\mathrm{OS}}\left(\omega_{\mathrm{ex}}\right)\right] /\left(2 G_{\mathrm{OS}}\right)=-k_{\mathrm{ex}} \eta \sin \varphi_{0} .}
\end{aligned}
$$

The reflected radiation, by having an effect on the oscillator OS, causes changes in the amplitude and phase of the oscillations relative to their stationary values $A_{\text {loc }}^{0}$ and $\varphi_{0}$. Since usually the relative levels of the reflected radiation and the synchronizing effect are small, so $\Gamma<<1$ and $k_{\text {ex }}<<1$, then we further assume that the amplitude $\Delta A$ and phase $\tilde{\varphi}$ variations in magnitude are fairly small, $|\Delta A|<<A^{0}{ }_{\text {loc }},|\tilde{\varphi}|<<1$. It should be borne in mind that the synchronizing effect can cause significant deviations of the stationary mode of a stand-alone oscillator as compared with the effects of reflected radiation, $k_{\mathrm{ex}}>>\Gamma$. Therefore, to describe the autodyne response of ILAD, we further linearized equations (3), (4) with respect to the stationary mode of synchronous oscillations described by expressions (5) and (6). After a series of transformations, we obtain a system of differential equations for small relative variations in amplitude $a=\Delta A / A^{0}{ }_{\text {loc }}$ and absolute changes in phase $\tilde{\varphi}$ in the form as follows:

$$
\begin{gathered}
\frac{\mathrm{d} a}{\mathrm{~d} t}+\frac{1}{\tau_{\mathrm{a}}} a+\frac{\operatorname{tg} \varphi_{0}}{\tau_{\varphi}} \tilde{\varphi}=\Gamma \frac{K_{\mathrm{a}}}{\tau_{\mathrm{a}}} \cos \delta(t, \tau), \\
\frac{\mathrm{d} \tilde{\varphi}}{\mathrm{d} t}+\frac{\gamma}{\tau_{\mathrm{a}}} a+\frac{1}{\tau_{\varphi}} \tilde{\varphi}=-\Gamma \frac{K_{\mathrm{a}}}{\tau_{\mathrm{a}}} \sin \delta(t, \tau)
\end{gathered}
$$

where $\varphi_{0}=-\left[\theta_{0}+\arcsin \chi_{\mathrm{n}}\right] ; \quad \theta_{0}=\arctan \gamma$ is the displacement angle; $\chi_{\mathrm{n}}=\left(\omega_{\mathrm{ex}}-\omega_{0}\right) / \Delta_{\mathrm{m}}$ is the normalized frequency 
detuning $\omega_{\mathrm{ex}}$ and the natural oscillation frequency $\omega_{0}$ of a stand-alone microwave oscillator; $\Delta_{\mathrm{m}}=k_{\mathrm{ex}} \omega_{0}\left(1+\gamma^{2}\right)^{1 / 2} / Q_{\mathrm{ex}}$ is the half-width of the synchronization band of the oscillator; $K_{\mathrm{a}}=\eta / \alpha$ is the autodyne gain factor; $\tau_{\mathrm{a}}=Q_{\mathrm{L}} /\left(\alpha \omega_{0}\right)$, $\tau_{\varphi}=Q_{\mathrm{ex}} /\left(k_{\mathrm{ex}} \omega_{0} \cos \varphi_{0}\right)$ are the time relaxation constants of amplitude and phase, respectively; $\gamma=\beta / \alpha$ is the oscillator non-isochronous factor; $\alpha=\left(A_{0} /\left(2 G_{\mathrm{OS}}\right)\right)\left(\partial G_{\mathrm{AE}} / \partial A\right)_{0}$ and $\beta=\left(A_{0} /\left(2 G_{\mathrm{OS}}\right)\right)\left(\partial B_{\mathrm{AE}} / \partial A\right)_{0}$ are the dimensionless parameters characterizing the strength of the limit cycle and nonisodromic of the oscillator, respectively.

The resulting system of equations (7), (8), in principle, describes the ILAD behavior implemented on the basis of any type of AE (tunnel diodes, Gunn diodes and avalanche-transit diodes, field-effect and bipolar transistors etc.).

\section{Analysis and Calculation of Autodyne Frequency Deviation of the ILAD}

In analyzing the autodyne frequency deviation of the ILAD, it makes sense to consider the general case of a moving location object, when $\tau \equiv \tau(t)$. Then the derivative of the phase changes will allow us to determine the dynamic changes in the frequency generation. To do this, under the condition of constant rate of movement, we write the phase incursion $\delta(t, \tau)$ as follows:

$$
\delta(t, \tau)=\omega_{\mathrm{ex}} \tau(t)=\Omega_{\mathrm{D}} t+\delta_{0}
$$

where $\Omega_{\mathrm{D}}=\omega_{\mathrm{ex}}(\mathrm{d} \tau / \mathrm{d} t)$ is the autodyne frequency signal equal to Doppler frequency; $\delta_{0}=\omega_{\mathrm{ex}} \tau_{0}$ is the initial phase of the Doppler signal, which we further assume to be zero. After transformation of the system (7), (8) in view of (9), we obtain the differential equation for $\tilde{\varphi}$ phase variations in the form:

$$
\begin{aligned}
& \frac{\mathrm{d}^{2} \tilde{\varphi}}{\mathrm{d} t^{2}}+\frac{\tau_{\mathrm{a}}\left(1+k_{\Omega}\right)}{k_{\Omega} \tau_{\mathrm{a}}^{2}} \frac{\mathrm{d} \tilde{\varphi}}{\mathrm{d} t}+\frac{1-\gamma \operatorname{tg} \varphi_{0}}{k_{\Omega} \tau_{\mathrm{a}}^{2}} \tilde{\varphi}= \\
& -\Gamma \frac{K_{\mathrm{a}}}{\tau_{\mathrm{a}}^{2}}[\sin (\Omega t)+(\gamma+\Omega) \cos (\Omega t)]
\end{aligned}
$$

where $\Omega=\tau_{\mathrm{a}} \Omega_{\mathrm{D}}$ is the normalized frequency; $k_{\Omega}=\tau_{\varphi} / \tau_{\mathrm{a}}=1 /\left(U_{\mathrm{ex}} \cos \varphi_{0}\right)$ is the coefficient characterizing the ratio of time constants $\tau_{\varphi}$ and $\tau_{\mathrm{a}} ; U_{\mathrm{ex}}=k_{\mathrm{ex}} K_{\mathrm{a}}$ is the relative level of the external synchronization signal with regard to its autodyne gain.

The general solution of the equation for dynamic changes in the phase $\tilde{\varphi}(t)$ and frequency $\tilde{\omega_{\mathrm{a}}}(t)=\mathrm{d}[\tilde{\varphi}(t)] / \mathrm{d} t$ of ILAD, after a series of elementary transformations, can be written as follows:

$$
\begin{aligned}
& \tilde{\varphi}(t)=-\left(\Gamma / k_{\mathrm{ex}}\right) H_{\varphi} \Phi_{\mathrm{din}}(\Omega) \sin \left[\Omega t+\theta_{\mathrm{din}}(\Omega)\right], \\
& \tilde{\omega}_{\mathrm{a}}(t)=-\left(\Gamma / k_{\mathrm{ex}}\right) H_{\varphi} D_{\mathrm{din}}(\Omega) \cos \left[\Omega t+\theta_{\mathrm{din}}(\Omega)\right]
\end{aligned}
$$

where $H_{\varphi}$ is the autodyne transfer coefficient of the ILAD phase:

$$
H_{\varphi}=\frac{\left(1+\gamma^{2}\right)^{1 / 2}}{\left(1-\gamma \operatorname{tg} \varphi_{0}\right) \cos \varphi_{0}}
$$

$\Phi_{\text {din }}(\Omega), D_{\text {din }}(\Omega)$ are the coefficients determining the formation of amplitude-frequency response (AFR) characteristics of the autodyne phase and frequency transfer coefficients in the dynamic mode ILAD:

$$
\begin{gathered}
\Phi_{\mathrm{din}}(\Omega)=\sqrt{w_{\mathrm{s}}^{2}(\Omega)+w_{\mathrm{c}}^{2}(\Omega)} / H_{\varphi} \cos \varphi_{0}, \\
D_{\mathrm{din}}(\Omega)=\Omega \Phi_{\mathrm{din}}(\Omega) ;
\end{gathered}
$$

$\theta_{\text {din }}(\Omega)$ is the displacement angle of the autodyne variations in the phase and frequency oscillations in the dynamic mode of ILAD:

$$
\theta_{\text {din }}(\Omega)=\operatorname{arctg}\left[w_{\mathrm{c}}(\Omega) / w_{\mathrm{s}}(\Omega)\right]
$$

$w_{\mathrm{s}}(\Omega), w_{\mathrm{c}}(\Omega)$ are the autodyne signal frequency-dependent 'sine' (index 's') and 'cosine' (index 'c') factors obtained in solving the equation (10):

$$
\begin{aligned}
& w_{\mathrm{s}}(\Omega)=\frac{\left(1-\gamma \operatorname{tg} \varphi_{0}-k_{\Omega} \Omega^{2}\right)+(\gamma+\Omega)\left(1+k_{\Omega}\right) \Omega}{\left(1-\gamma \operatorname{tg} \varphi_{0}-k_{\Omega} \Omega^{2}\right)^{2}+\left(1+k_{\Omega}\right)^{2} \Omega^{2}}, \\
& w_{\mathrm{c}}(\Omega)=\frac{(\gamma+\Omega)\left(1-\gamma \operatorname{tg} \varphi_{0}-k_{\Omega} \Omega^{2}\right)-\left(1+k_{\Omega}\right) \Omega}{\left(1-\gamma \operatorname{tg} \varphi_{0}-k_{\Omega} \Omega^{2}\right)^{2}+\left(1+k_{\Omega}\right)^{2} \Omega^{2}} .
\end{aligned}
$$

From the expressions (11) and (12), it can be seen that the maximum changes in phase $\tilde{\varphi}$ and frequency $\tilde{\omega}_{\mathrm{a}}$ are directly proportional to the $\Gamma / k_{\mathrm{ex}}$ ratio. In addition, the amplitudes of the variations, as can be seen from (11) and (12), are proportional to the magnitude of the transfer coefficient $H_{\varphi}$ of the autodyne phase changes. In this case, the value of this coefficient determines the 'initial' deviation values of the phase $\tilde{\varphi}$ and frequency $\tilde{\omega}_{\mathrm{a}}$ in the low-frequency region $\Omega$, where the strong inequality $2 \pi / \Omega_{\mathrm{D}}>>\tau_{\mathrm{a}}$ is performed.

In turn, the value $H_{\varphi}$ largely depends on the synchronization conditions of the microwave oscillator which are determined through the angle $\varphi_{0}$ by the magnitude and sign of the normalized detuning sign $\chi_{\mathrm{n}}$. From the diagrams $H_{\varphi}\left(\chi_{\mathrm{n}}\right)$ in Fig. 2 calculated according to (13), it can be seen that in the center of the synchronization band, the transfer coefficient $H_{\varphi}$ has the lowest value, and its rapid growth can be observed towards the edges of the synchronization band. At the same time, the sign and values of the nonisochronous factor $\gamma$ of the oscillator virtually do not affect the course of the curves $H_{\varphi}\left(\chi_{\mathrm{n}}\right)$. Another important conclusion follows from (15): the autodyne frequency deviation of the ILAD takes place only in dynamics, i.e. in the case of moving a reflecting object, when $\Omega \neq 0$.

An analysis of the AFR coefficients $\Phi_{\text {din }}(\Omega)$ and $D_{\text {din }}(\Omega)$ of ILAD will be performed numerically by introduc- 


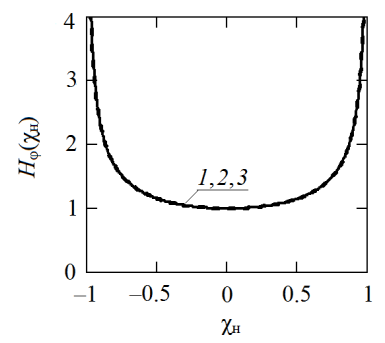

Fig. 2. Dependency diagrams calculated for different values of factor $\gamma \cdot \gamma=1.5$ (curve 1 ), $\gamma=0$ (curve 2), $\gamma=-2.5$ (curve 3).

ing the variations of various system parameters. Figure 3 shows the calculation results according to (14) and (15) by taking into account (17), (18) and (13) of the family of AFR diagrams $\Phi_{\operatorname{din}}(\Omega)$ and $D_{\operatorname{din}}(\Omega)$ obtained for the isochronous oscillator $(\gamma=0)$, at different levels of the synchronizing signal. Figure 4 shows the AFR diagrams $D_{\text {din }}(\Omega)$ for various cases of an isochronous oscillator and non-isochronous oscillator $(\gamma \neq 0)$ with variations in the conditions of synchronization of the oscillator. It should be noted that in figures, frequency $\Omega=1$ corresponds to the critical frequency $\Omega_{\text {cr }}^{\mathrm{a}}$ on the level of $1 / 2^{1 / 2}$ AD without synchronization, $\Omega^{\mathrm{a}}{ }_{\mathrm{cr}}=1 / \tau_{\mathrm{a}}$. In practice, the value $\tau_{\mathrm{a}}$, for example, in the $8-\mathrm{mm}$ wavelength range for oscillators on the Gunn diodes can be in the range of $1 \mathrm{~ns}$ [31]. In this case, the cut-off frequency can reach $\Omega_{\mathrm{cr}}^{\mathrm{a}} \approx 2 \pi \times 150 \times 10^{6}$.

As follows from the diagrams of Fig. 3(a) as well as the calculation results for other values of the parameters $k_{\Omega}$, $U_{\mathrm{ex}}, \chi_{\mathrm{n}}$ and $\gamma$ AFR of the ILAD coefficients $\Phi_{\mathrm{din}}(\Omega)$ are the monotonic functions of frequency. When the frequency $\Omega$ of the critical value $\Omega^{\varphi}{ }_{\mathrm{cr}}=1 / \tau_{\varphi}$ is exceeded by level $1 / 2^{1 / 2}$, the $\Phi_{\text {din }}(\Omega)$ coefficient value drops sharply. The drop in AFR can be explained by the inertia of the oscillator synchronization, during which the ILAD oscillation phase does not have time to respond to rapid variations in the phase incursion $\delta(t, \tau)$ of the reflected radiation.

The diagrams in Fig. 3(b) and Fig. 4 show that in the case of a fixed object, when $\Omega=0$, the coefficient $D_{\text {din }}(\Omega)=0$ and there is no frequency deviation. If a location object is moved, when the frequency of the autodyne signal is in the region of low frequencies, where $\Omega<\Omega^{\varphi}$ cr, the value $D_{\text {din }}(\Omega)$ increases in proportion to the frequency $\Omega$

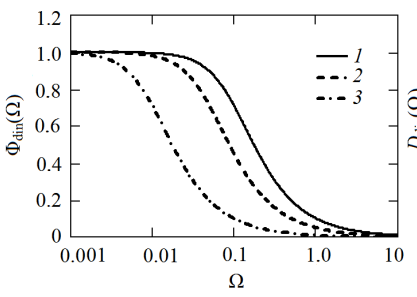

(a)

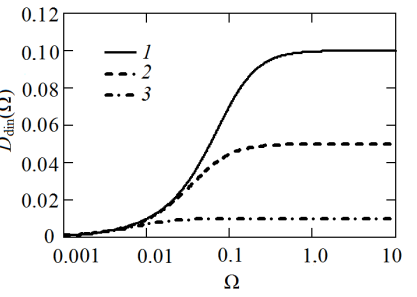

(b)
Fig. 3. Diagrams of the AFR coefficients $\Phi_{\text {din }}(\Omega)$ (a) and $D_{\text {din }}(\Omega)$ (b) calculated for the case $\gamma=0, \chi_{\mathrm{n}}=0$ and with variations in the level of external exposure $U_{\text {ex }}$ : $U_{\mathrm{ex}}=0.1$ (curves 1$), U_{\mathrm{ex}}=0.05$ (curves 2$), U_{\mathrm{ex}}=0.01$ (curves 3).

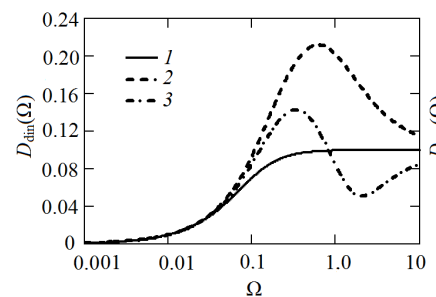

(a)

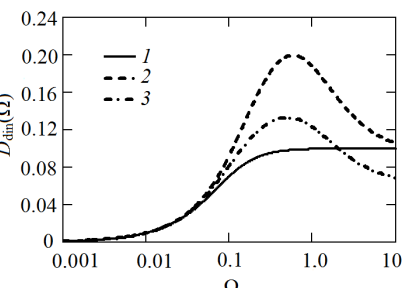

(b)
Fig. 4. Diagrams of AFR $D_{\mathrm{din}}(\Omega)$ calculated at $U_{\mathrm{ex}}=0.1$ for the case (a) at $\chi_{\mathrm{n}}=0$ and variation $\gamma \cdot \gamma=0$ (curve 1 ), $\gamma=1.5$ (curve 2), $\gamma=-1.5$ (curve 3) and for the case (b) at $\gamma=1.5$ and initial detuning variations $\chi_{\mathrm{n}}: \chi_{\mathrm{n}}=0$ (curve 1$), \chi_{\mathrm{n}}=0.4($ curve 2$), \chi_{\mathrm{n}}=0.8($ curve 3$)$.

and, accordingly, the rate of movement of the object. In the high-frequency region, where $\Omega>\Omega^{\varphi}$ cr, due to the synchronization inertia, the autodyne deviation of the $\Delta \omega_{\text {ILAD }}$ frequency of the isochronous oscillator (see Fig. 3(b)) does not change significantly. In the case of a non-isochronous oscillator (see graphics in Fig. 4), in the region comparable with $\Omega^{\varphi}{ }_{\text {cr }}$ of $\Omega$ frequencies, there is an uneven AFR. Moreover, the type of the AFR coefficient $D_{\text {din }}(\Omega)$ is largely determined by the synchronization conditions (the value of the parameters $U_{\text {ex }}$ and $\chi_{\mathrm{n}}$ ) as well as the magnitude and sign of the non-isochronism coefficient $\gamma$.

The reason for the unevenness of the AFR coefficient $D_{\text {din }}(\Omega)$ in the frequency domain $\Omega>\Omega^{\varphi}$ cr is associated with the internal 'interference' of the components of the autodyne response due to a change in the phase $\tilde{\varphi}(t)$ of oscillations. The first component is associated with the conversion of $a(t)$ amplitude variations into variations in the phase $\tilde{\varphi}(t)$ of the oscillations of the microwave oscillator (see the second member of the left-hand side (8)). The second component is subject to variations in the phase incursion $\delta(t, \tau)$ of the reflected microwave radiation (see the right-hand side of (8)). The dynamic shifts of phase $\theta_{\text {din }}(\Omega)$ of the autodyne response depending on the frequency $\Omega$, as shown by calculations according to (16), cause variations in the phase relationships between the additive components. Therefore, the resulting response in respect of the changed frequency $\tilde{\omega_{\mathrm{a}}}(t)$ increases at those frequencies, at which both components are in-phase and is attenuated at frequencies with the antiphase components.

It should be noted that the operating frequencies of the $\mathrm{AD}$ signals are usually in the low frequency region, where $\Omega \in 0 \ldots 0.01$. The diagrams of Fig. 3(a) show that $\Phi_{\text {din }}(\Omega)=1$ can be taken in the AFR area. In addition, we simplify (13) assuming a transmission coefficient $H_{\varphi} \approx 1 / \cos \varphi_{0}$ in the synchronization band. Then the autodyne deviation of the frequency of ILAD in (12) can be calculated by the following simplified formula: $\Delta \omega_{\mathrm{ILAD}} \approx \Gamma \Omega_{\mathrm{D}} /\left(k_{\mathrm{ex}} \cos \varphi_{0}\right)$. To compare the values of the autodyne deviation of the frequency of ILAD and ordinary $\mathrm{AD} \Delta \omega_{\mathrm{AD}} \approx \Gamma \omega_{0} / Q_{\mathrm{ex}}$ [16] with the same level of the reflected microwave radiation, we take their ratio and, in addition, take into account the formula for calculating the Doppler frequency $\Omega_{\mathrm{D}}=2 V_{\mathrm{r}} \omega_{0} / c$ : 


$$
\frac{\Delta \omega_{\mathrm{AD}}}{\Delta \omega_{\mathrm{ILAD}}} \approx \frac{\omega_{0} k_{\mathrm{ex}} \cos \varphi_{0}}{\Omega_{\mathrm{D}} Q_{\mathrm{ex}}}=\frac{c k_{\mathrm{ex}} \cos \varphi_{0}}{2 Q_{\mathrm{ex}} V_{\mathrm{r}}}
$$

where $V_{\mathrm{r}}$ is the radial component of the relative velocity of the radar object; $c$ is the microwave radiation propagation velocity.

From the formula (19) it can be seen that by varying the parameters $k_{\mathrm{ex}}$ and $\varphi_{0}$ it is possible to choose the required values of the ILAD frequency deviation. The calculation by the formula for the values of $Q_{\mathrm{ex}}=150$, $k_{\mathrm{ex}}=0.1, V_{\mathrm{r}}=1000 \mathrm{~m} / \mathrm{s}$ and $\cos \varphi_{0}=1$ gives the gain from the use of ILAD $\Delta \omega_{\mathrm{AD}} / \Delta \omega_{\mathrm{ILAD}}=100$. Thus, the external synchronization of the oscillator significantly reduces the amount of frequency autodyne deviation, and thus effectively solves the problem of combating the anharmonic distortion of AD signals.

\section{Experimental Results}

Experimental research into ILAD was carried out by the example of a "Tigel-08M", manufactured with the hybrid integrated technology based on the Gunn planar diode (see Fig. 5). The module package is made in the form of two plates, between which the diode insertion is included with the thickness $0.5 \mathrm{~mm}$ from the polycor. The split resonator topology in the half-wavelength is made on this diode insertion. Chips of the Gunn diode oscillator and the detector diode with the Schottky barrier are mounted across this split. There is a hole of circular shape for the microwave emission in the center of the face plate of the package. There is the adjusting screw for frequency generation control in the back plate of three packages. The supply voltage of the module is $4 \mathrm{~V}$ at a current consumption of about $0.2 \mathrm{~A}$. The oscillation frequency is $37.5 \mathrm{GHz}$, the output power is $15 \mathrm{~mW}$.

The block-diagram of the experimental workbench is shown in Fig. 6 [26]. The examined AD is connected with the Doppler simulator DS by the waveguide path WL with the length of $12 \mathrm{~m}$. The variable attenuator At and the directional coupler DC are also in this section. The first side arm of the DC coupler is intended for connection of the synchronizing signal from the MO oscillator.

The second side arm of the DC coupler is connected to the input of frequency converter FC, which transits the $\mathrm{AD}$ emission spectrum into the frequency $1.0 \mathrm{GHz}$. The output signal of FC passes to the frequency discriminator FD to obtain the signal $u_{\omega}(t)$ proportional to the autodyne variations of the generated frequency.

The photo-picture of the external view and the construction sketch of the Doppler simulator are shown in Fig. 7. The segment of the rectangular waveguide 1, in which along the middle of the wide wall of the internal side of the half-circle, there is a split 2, which is curved in the half-circle, is the base of the simulator (see Fig. 7(b)) The pin reflectors 3 mounted on the disk 4 are introduced in the

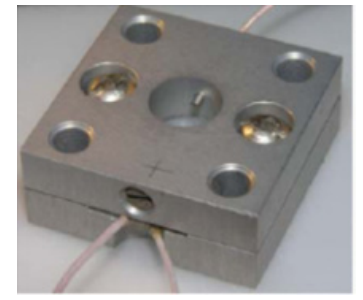

(a)

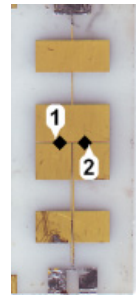

(b)
Fig. 5. The external view of the autodyne module «Tigel$08 \mathrm{M} »(\mathrm{a})$, the topology of the diode insertion (b): 1 is the chip of the planar Gunn diode; 2 is the chip of the Schottky diode.

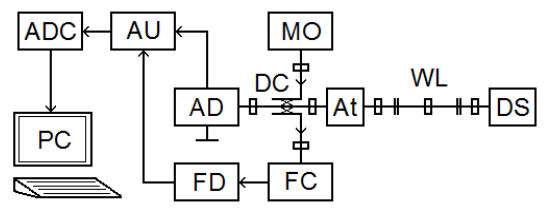

\begin{tabular}{llll} 
& \multicolumn{3}{c}{ Abbreviations meaning } \\
AD & autodyne & FC & frequency carrier \\
At & attenuator & FD & frequency discriminator \\
DC & directional coupler & AU & analog unit \\
DS & Doppler simulator & ADC & analog-to-digital converter \\
WL & waveguide line & PC & personal computer \\
MO & measuring oscillator & &
\end{tabular}

Fig. 6. Block diagram experimental setup.

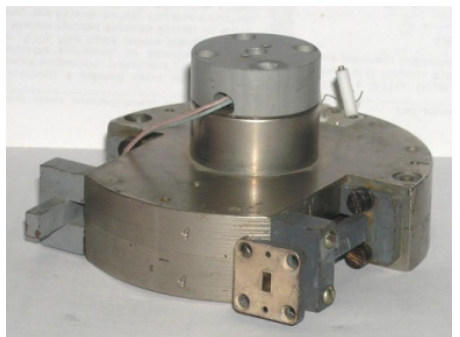

(a)

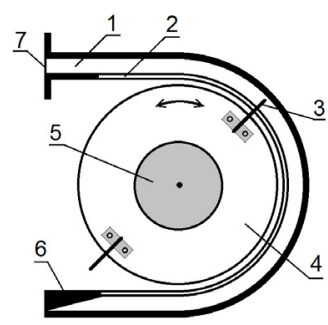

(b)
Fig. 7. External view (a) and construction sketch (b) of the Doppler signal simulator: 1 is the rectangular waveguide; 2 is the split in the wide wall of the waveguide; 3 is the pin reflector; 4 is the disk; 5 is the electrical motor; 6 is the absorbing load; 7 is the input-output of the simulator.

waveguide cavity through this split. The disk is mounted of the electrical motor 5 axis. At that, in the one of the ends of the curved waveguide, the absorbing load 6 in mounted, while the second end is the input-output 7 of the simulator.

Signals $u_{\mathrm{a}}(t)$ from the output of the detector Schottky diode and $u_{\omega}(t)$ from the output of the frequency discriminator FD are applied for amplification and filtering into the analog block AU. From the AU output, they passed to inputs of analog entry channels of the module NI-9205 from the National Instruments in the structure of DAQ9172 platform of the block of analog-to-digital conversion (ADC). Then, digitized signals pass via the USB interface into the personal computer PC for processing with the help of the virtual device created in LabView 8.6.

A variable attenuator is used in the waveguide duct to set the attenuation value of $30 \mathrm{~dB}$, at which the autodyne 


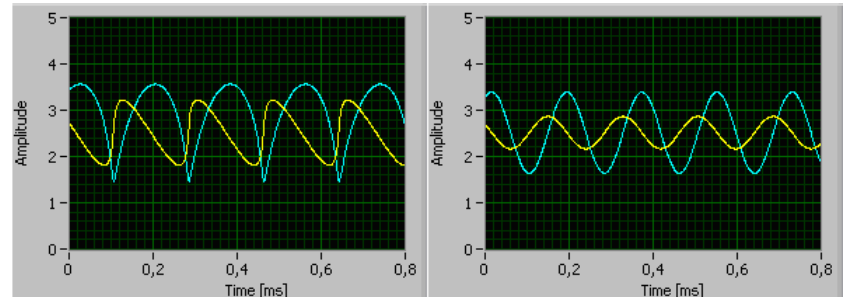

(a)

(b)

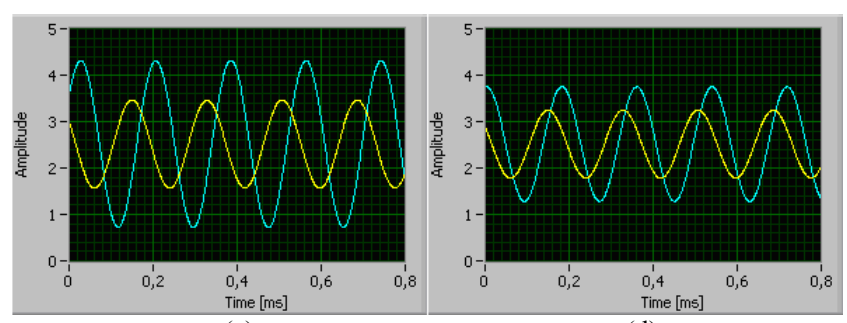

(c)

(d)

Fig. 8. Signal oscillograms (blue track) and $u_{\omega}(t)$ (yellow track) of ILAD on the Gunn diode obtained in the absence of synchronization (a); and when available for cases where $\chi_{n}=0$ (b), $\chi_{n}=-0.4$ (c) and $\chi_{n}=0.4$ (d).

frequency deviation is $\Delta \omega_{\mathrm{AD}} \approx 2 \pi \times 1.3 \times 10^{6}$. Under these conditions, the calculated value of the parameter $C_{\mathrm{FB}} \approx 0.8$ is provided. Usually, with such a value $C_{\mathrm{FB}}$, the signals of AD have a noticeable level of anharmonic distortions which can be confirmed by the signal oscillograms $u_{\mathrm{a}}(t)$ and $u_{\omega}(t)$ presented in Fig. 8. At that, the output signal of MO with the value about $1 \mathrm{~mW}$ provided the half-width of the AD synchronization band $\Delta_{m} \approx 2 \pi \times 50 \times 10^{6}$.

The remaining oscillograms in Fig. $8(\mathrm{~b}, \mathrm{c}, \mathrm{d})$ are obtained when a synchronization signal is applied to the oscillator module and at various values of the normalized detuning $\chi_{n}$. From the oscillograms it can be seen that the ILAD signals $u_{\mathrm{a}}(t)$ and $u_{\omega}(t)$ are virtually harmonic. The value $\Delta \omega_{\mathrm{ILAD}} \approx 2 \pi \times 0.5 \times 10^{3}$ of the autodyne frequency deviation of the module is obtained, i.e. the payoff is $\Delta \omega_{\mathrm{AD}} / \Delta \omega_{\mathrm{ILAD}}=2600$. It should be noted that in the middle of the sync band, the frequency deviation of the ILAD is the smallest (see Fig. 8(b)), and as it approaches its edges, it increases asymmetrically (see Fig. 8(c, d)). The velocity of movement of the reflector of the Doppler signal simulator in performing the described experiments was maintained constant at about $20 \mathrm{~m} / \mathrm{s}$. An increase in the reflector velocity was accompanied by a corresponding increase in the frequency of the signals $u_{\mathrm{a}}(t)$ and, as well as the amplitude of the signal $u_{\omega}(t)$.

Thus, the experimental research results have qualitatively confirmed the adequacy of the developed mathematical ILAD model with respect to the description of autodyne frequency deviation as well as the conclusions obtained in the theoretical analysis.

\section{Conclusions}

As a result of the performed ILAD research, the basic relations for calculating its autodyne frequency deviation are obtained for the conditions of both slow movements of a radar object and at high velocities, when the autodyne signal period is comparable with the values of the autodyne response constant values. It has been established that the value of the autodyne frequency deviation of ILAD in the whole range of velocities of movement of radar objects that exist in practice is directly proportional to the frequency of the Doppler signal. In this case, the proportionality coefficient can be adjusted by choosing the conditions of the oscillator synchronization, the level of the synchronizing effect and initial detuning between the frequencies of the external oscillator and the natural frequency $\mathrm{AD}$ within the synchronization band.

It is shown that in the center of the synchronization band the value of the autodyne frequency deviation is the lowest; and when the signal frequency of the synchronizing oscillator is reset to its edges, deviation rapidly increases. At the same time, the absolute values of the autodyne deviation of the ILAD oscillation frequency at the same level of the reflected microwave radiation are much lower (by tens of $\mathrm{dB}$ ) than the AD deviation without external synchronization. Due to the reduction of the deviation, the non-linearity of the phase progression of the reflected microwave radiation, the feedback parameter of the autodyne system 'oscillator - radar object', and finally, the degree of the anharmonic distortion of signals are reduced accordingly.

From the results of the work, a conclusion should also be drawn that the development of autodyne hybrid integrated modules of the millimeter range with the frequency synchronization from an additional low-power oscillator has a potential. In this case, of particular interest is the use in the synchronization oscillator of low-current mesa-planar Gunn diodes [32].

\section{References}

[1] HINMAN, W. S., BRUNETTI, C. Radio proximity fuze design. Part of Journal of Research of the National Bureau of Standards, 1946, vol. 37, RP1723, p. 1-13. https://nvlpubs.nist.gov/nistpubs/ jres/37/jresv37n1p1_A1b.pdf

[2] PANTOJA, F. R., CALAZANS, E. T. Theoretical and experimental studies of gain compression of millimeter-wave selfoscillating mixers. IEEE Transactions on Microwave Theory and Techniques, 1985, vol. 33, no. 3, p. 181-186. DOI: 10.1109/TMTT.1985.1132979

[3] CLAASSEN, M. Self-mixing oscillators. In: Luy, J. F., Russer, P. (eds.) Silicon-Based Millimeter-Wave Devices. Springer Series in Electronics and Photonics, vol. 32. Berlin, Heidelberg (Germany): Springer, 1994. p. 215-239. DOI: 10.1007/978-3-642-79031-7_6

[4] NYGREN, T., SJOLUND, A. Sensitivity of Doppler radar with self-detecting diode oscillators. IEEE Transactions on Microwave Theory and Techniques, 1974, vol. 22, no. 5, p. 494-498. DOI: 10.1109/TMTT.1974.1128268

[5] NAGANO, S., AKAIWA, Y. Behavior of a Gunn diode oscillator with a moving reflector as a self-excited mixer and a load variation detector. IEEE Transactions on Microwave Theory and Techniques, 1971, vol. 19, no. 2, p. 906-910. DOI: 10.1109/TMTT.1971.6373339 
[6] TAKAYAMA, Y. Doppler signal detection with negative resistance diode oscillators, IEEE Transactions on Microwave Theory and Techniques, 1973, vol. 21, no. 2, p. 89-94. DOI: 10.1109/ TMTT.1973.1127929

[7] KITTIPUTE, K., SARATAYON, P., SRISOOK, S., et al. Homodyne detection of short-range Doppler radar using a forced oscillator model. Scientific Reports, 2017, vol. 7, p. 1-13. DOI: $10.1038 /$ srep43680

[8] KOMAROV, I. V., SMOLSKIY, S. M. Fundamentals of ShortRange FM Radar. Norwood (MA, USA): Artech House, 2003. ISBN: 978-1580531108

[9] VOTOROPIN, S. D., ZAKARLYUK, N. M., NOSKOV, V. YA., et al. On principal impossibility of auto-synchronization of an autodyne by radiation reflected from a moving target. Russian Physics Journal, 2007, vol. 50, no. 9, p. 195-206. DOI: $10.1007 / \mathrm{s} 11182-007-0132-2$

[10] USANOV, D. A., SKRIPAL, AL. V., SKRIPAL, AN. V., et al. A microwave autodyne meter of vibration parameters. Instruments and Experimental Techniques, 2004, vol. 47, no. 5, p. 689-693. DOI: 10.1023/B:INET.0000043882.16801.3a

[11] Alidoost, S. A., SADEGHZADE, R., FATEMI, R. Autodyne system with a single antenna. In 11th International Radar Symposium (IRS-2010). Vilnius (Lithuania), 2010, vol. 2, p. 406-409. ISBN: 978-9955-690-18-4

[12] USANOV, D. A., POSTElGA, A. E. Reconstruction of complicated movement of part of the human body using radio wave autodyne signal. Biomedical Engineering, 2011, vol. 45 , no. 1, p. 6-8. DOI: $10.1007 / \mathrm{s} 10527-011-9198-9$

[13] ERMAK, G. P., POPOV, I. V., VASILIEV, A. S., et al. Radar sensors for hump yard and rail crossing applications. Telecommunication and Radio Engineering, 2012, vol. 71, no. 6 , p. 567-580. DOI: 10.1615/TelecomRadEng.v71.i6.80

[14] KIM, S., KIM, B.-H., YOOK, J.-G., et al. Proximity vital sign sensor using self-oscillating mixer. In URSI Asia-Pacific Radio Science Conference (URSI AP-RASC). Seoul (South Korea), 2016, p. 1446-1448. DOI: 10.1109/URSIAP-RASC.2016.7601402

[15] USANOV, D. A., SKRIPAL, A. V., KASHCHAVTSEV, E. O., et al. Acceleration measurements upon micro- and nanodisplacements of an object using the autodyne signal of a semiconductor laser with allowance for the external optical feedback. Technical Physics, 2013, vol. 58, no. 7, p. 1083-1085. DOI: $10.1134 / \mathrm{S} 1063784213070244$

[16] NOSKOV, V. YA., ERMAK, G. P. Measurement errors and dynamic range of autodyne vibration meters. Telecommunication and Radio Engineering, 2014, vol. 73, no. 20, p. 1843-1861. DOI: 10.1615/TelecomRadEng.v73.i20.50

[17] NOSKOV, V. YA., IGNATKOV, K. A., CHUPAHIN, A. P. Application of two-diode autodynes in devices for radiowave control of product dimensions. Measurement Techniques, 2016, vol. 59, no. 7, p. 715-721. DOI: 10.1007/s11018-016-1035-9

[18] NOSKOV, V. YA., IGNATKOV, K. A. Peculiarities of noise characteristics of autodynes under strong external feedback. Russian Physics Journal, 2013, vol. 56, no. 12, p. 1445-1460. DOI: $10.1007 / \mathrm{s} 11182-014-0198-6$

[19] VOTOROPIN, S. D., NOSKOV, V. YA., SMOLSKIY, S. M. An analysis of the autodyne effect of a radio-pulse oscillator with frequency modulation. Russian Physics Journal, 2008, vol. 51, no. 7 , p. $750-759$. DOI: $10.1007 / \mathrm{s} 11182-008-9105-3$

[20] NOSKOV, V. YA., IGNATKOV, K. A., CHUPAHIN, A. P., et al. Signals of autodyne sensors with sinusoidal frequency modulation. Radioengineering, 2017, vol. 26, no. 4, p. 1182-1190. DOI: $10.13164 /$ re.2017.1182
[21] NOSKOV, V. YA. Peculiarities of self-oscillations of a biharmonic autodyne receiver under effect of reflected radiation. Izvestiya VUZ: Radioelektronika, 1991, vol. 34, no. 11, p. 44-50. (in Russian)

[22] NOSKOV, V. YA. Autodyne effect in multifrequency oscillators. Radiophysics and Quantum Electronics, 1992, vol. 35, no. 9-10, p. 503-508. DOI: 10.1007/BF01044970

[23] MOROSANOV, S. A., SMOLSKIY, S. M., FILITSINA, YU. A. Push-pull transistor oscillators and autodyne. Radiotekhnika $i$ elektronika [Radio Engineering and Electronics], 1982, vol. 27, no. 4, p. 764-769. (in Russian)

[24] NOSKOV, V. YA. A double-diode autodyne transceiver. Instruments and Experimental Techniques, 2015, vol. 58, no. 3, p. 505-509. DOI: $10.1134 / \mathrm{S} 0020441215030240$

[25] NOSKOV, V. YA., IGNATKOV, K. A., SMOLSKIY, S. M. Analysis of signals of stabilized autodynes. Telecommunication Sciences, 2011, vol. 2, no. 1, p. 5-16. ISSN: 2219-9454

[26] NOSKOV, V. YA., IGNATKOV, K. A. Noise characteristics of autodynes with frequency stabilization by means of an external high-Q cavity. Journal of Communications Technology and Electronics, 2016, vol. 61, no. 9, p. 1052-1063. DOI: $10.1134 / \mathrm{S} 1064226916090102$

[27] TERESCHENKO, A. F. Impact of the reflected signal on a synchronized klystron oscillator. Elektronnaya tehnika. Seriya 1. SVCh-tehnika [Electronic Technique. Series 1. UHF Technique], 1966, no. 2, p. 67-78. (in Russian)

[28] ARTEMENKOV, S. L., SMOLSKIY, S. M. Autodyne properties of synchronized transistor oscillators. Metody $i$ ustroistva formirovaniya i obrabotki radiosignalov [Methods and Devices for the Formation and Processing of Radio Signals], Moscow, Trudy MEI, 1982, no. 579, p. 81-86. (in Russian)

[29] ARTEMENKOV, S. L., SMOLSKIY, S. M. Stabilization of the transistor autodyne frequency by additional clock signal. Sovremennie problemi stabilizacii chastoti [Current Problems of Frequency Stabilization], Moscow, Trudy MEI, 1983, no. 8, p. 30-35. (in Russian)

[30] KUROKAVA, K. Injection locking of microwave solid-state oscillators. Proceedings of the IEEE, 1973, vol. 61, no. 10, p. 1386-1410. DOI: 10.1109/PROC.1973.9293

[31] NOSKOV, V. YA., IGNATKOV, K. A. Dynamic autodyne and modulation characteristics of microwave oscillators. Telecommunication and Radio Engineering, 2013, vol. 72, no. 10, p. 919-934. DOI: 10.1615/TelecomRadEng.v72.i10.70

[32] VOTOROPIN, S. D. Self-oscillating mixer EHF of the mezaplanar Gunn diodes. In 16th International Crimean Conference on Microwave and Telecommunication Technology. Sevastopol (Crimea, Ukraine), 2006, vol. 1, p. 148-149. DOI: 10.1109/CRMICO.2006.256336

\section{About the Authors ...}

Vladislav Ya. NOSKOV was born in 1948. He graduated from the Radio Engineering Faculty, Tomsk Institute of Automated Control Systems and Radio Electronics in 1973. He received the Ph.D. degree in 1985, and the Dr. Sci. degree in 1995. At present, he is a professor of the Dept. of Radioelectronics and Telecommunications, Inst. of Radioelectronics and Information Technology (IRITRTF), Ural Federal University (URFU). He authored more than 350 scientific publications and 18 patents. 
Kirill A. IGNATKOV was born in 1988. He graduated from the Institute of Radioelectronics and Information Technologies of the Ural Federal University in 2010. Ph.D. thesis was defended in 2014. At present, he is a $\mathrm{PhD}$, assistant professor of the Department of Radioelectronics and Telecommunications, Institute of Radioelectronics and Information Technology - RTF (IRIT-RTF), Ural Federal University (URFU). He authored more than 150 scientific publications and 5 patents.
Kirill D. SHAIDUROV was born in 1992. He graduated from the Institute of Radioelectronics and Information Technologies of the Ural Federal University in 2014 and currently is the 4nd-year PhD-student at Technologies and Communication Means Dept. At present, he is an assistant of the Department of Radioelectronics and Communications, Institute of Radioelectronics and Information Technology - RTF (IRIT-RTF), Ural Federal University (URFU). He authored 9 scientific publications. 